\title{
Efecto de la concentración y presión sobre la elevación del punto de ebullición de pasta de tomate (Lycopersicon esculentum Mill.)
}

\author{
Effect of concentration and pressure on the boiling point \\ elevation of tomato paste (Lycopersicon esculentum Mill.)
}

\section{Efeito da concentração e pressão na elevação do ponto de ebulição de concentrado de tomate (Lycopersicon esculentum Mill.)}

\author{
Fabián Alberto Ortega Quintana ${ }^{1 *}$; Yeni Lorena Isaza Maya²; Tammy Tirado Medrano2; \\ Everaldo Joaquín Montes Montes ${ }^{2}$
${ }^{1}$ Facultad de Minas. Universidad Nacional de Colombia. Carrera 80 No 65-223-Núcleo Robledo. Medellín, Colombia.
${ }^{2}$ Facultad de Ingeniería. Universidad de Córdoba. Carrera 6 No 76-103. Montería, Colombia. \\ *faortegaqui@unal.edu.co; ingenierofabianortega@gmail.com
}

Fecha Recepción: 14 de febrero de 2015 Fecha Aceptación: 19 de junio de 2015

\begin{abstract}
Resumen
Las propiedades térmicas de las pulpas concentradas juegan un papel importante en el diseño y optimización de evaporadores de múltiple efecto, entre estas propiedades se encuentra la elevación del punto de ebullición. En esta investigación se determinó la elevación del punto de ebullición de pastas de tomate (Lycopersicon esculentum Mill.) de las variedades colombianas Chonto, Milano y Río Grande variando las concentraciones de sólidos solubles desde 5 hasta $35^{\circ}$ Brix y las presiones de vacío desde 49,33 hasta 949,26 mbar. Cada tratamiento fue realizado por triplicado para cada variedad de tomate y se evaluó el ajuste de los datos obtenidos a los modelos matemáticos: Dühring, Antoine y Crapiste-Lozano. La concentración, la presión, la variedad de tomate y las diferentes interacciones de estas variables tuvieron efecto estadísticamente significativo al 5\% de significancia en la temperatura de ebullición de las pastas de tomate. Finalmente, para las tres variedades de tomate se encontró un buen ajuste de los datos experimentales a los diferentes modelos matemáticos.
\end{abstract}

Palabras clave: regla de Dühring, Crapiste y Lozano, Antoine, evaporadores, pasta de tomate.

\begin{abstract}
The thermal properties of concentrated pulps have an important role in the design and optimization of multiple effect evaporators; these properties include the boiling point elevation. In this research work, it was determined the boiling point elevation of tomato paste (Lycopersicon esculentum Mill.) of the Colombian varieties Chonto, Milano and Río Grande with concentrations of soluble solids between 5 and $35^{\circ}$ Brix and vacuum pressures between 49,33 and 949,26 mbar. The treatment was performed in triplicate for each variety of tomato and was assessed the adjustment to experimental data of the mathematical models: Dühring, Antoine and Crapiste-Lozano. The concentration, pressure, tomato variety and the different interactions of these variables had statistically significant effect at the $5 \%$ level in the boiling temperature of tomato paste. Finally, it was found a good fit of different mathematical models to experimental data of the three varieties of tomatoes.
\end{abstract}

Keywords: Dühring's rule, Crapiste and Lozano, Antoine, evaporators, tomato paste. 


\section{Resumo}

As propriedades térmicas de suspensões concentradas desempenha um papel importante no desenho e optimização de evaporadores de efeito múltiplo, entre estas propriedades é a elevação do ponto de ebulição. Nesta pesquisa, a elevação do ponto de ebulição de concentrado de tomate (Lycopersicon esculentum Mill.) de variedades colombianos Chonto, Milano e Río Grande foi determinada por diferentes concentrações de sólidos solúveis entre 5 e $35^{\circ}$ Brix e diferentes pressões de vácuo entre 49,33 e $949,26 \mathrm{mbar}$. Cada tratamento foi realizado em triplicata para cada variedade de tomate e os dados foram ajustados para modelos matemáticos: Dühring, Antoine e Crapiste-Lozano. Concentração, pressão, a variedade de tomate e as diferentes interacções entre estas variáveis teve efeito estatisticamente significativo no nível de $5 \%$ no ponto de ebulição do concentrado de tomate. Finalmente, para as três variedades de tomates um bom ajuste dos dados experimentais dos diferentes modelos matemáticos foram encontrados.

Palabras-chave: regra Dühring, Crapiste e Lozano, Antoine, evaporadores, concentrado de tomate.

\section{Introducción}

El tomate (Lycopersicon esculentum Mill.) es un fruto más importante en muchos países del mundo. Su cultivo está difundido a todos los continentes y en muchos casos representa una de las principales fuentes de vitaminas y minerales para la población. Si bien se cultiva tomate en más de cien países, tanto para consumo fresco como para industria, los diez principales productores concentran más del $70 \%$ del total mundial. Colombia ocupa el puesto número 36 en la producción mundial de tomate con una producción de 532425 toneladas para el año 2012, con un valor estimado de comercio de 516 mil millones de pesos [1].

El tomate es considerado como un fruto con alto contenido de agua, por lo tanto es muy perecedero, las pérdidas post-cosecha representan aproximadamente un $35 \%$ del total de las cosechas recolectadas debido a que su período de vida es muy corto luego que empieza su maduración. Uno de los principales usos industriales del tomate es la obtención de pasta mediante la concentración de sus sólidos solubles, de modo que se reduzca la cantidad de agua disponible para las reacciones de deterioro propias del tomate. Esta concentración generalmente se realiza en equipos al vacío, como los evaporadores de múltiple efecto, en donde se controlan las variables del proceso para conseguir mejores características del producto final [2].

Para el modelamiento matemático, diseño, optimización, control y operación de evaporadores de múltiple efecto se necesita información sobre la elevación del punto de ebullición que se presenta cuando un soluto no volátil es disuelto en determinado solvente y la temperatura de ebullición de la solución es siempre mayor que la temperatura del solvente puro [3]. Normalmente, para el caso particular de soluciones ideales, la elevación del punto de ebullición es predicha por la ley de Raoult, la cual establece que con un soluto no volátil, la solución entrará en ebullición cuando la presión parcial del agua se iguale con la presión del sistema. Para que esto ocurra es necesaria que la solución sea calentada hasta una temperatura superior a la temperatura de ebullición del agua pura [4]. La elevación del punto de ebullición puede ser expresada, a partir de la ley de Raoult, como:

$$
\Delta T_{B}=T-T_{w}=\frac{-T_{w}}{1+\left(\frac{\Delta H_{v}}{R T_{w} \operatorname{Ln}\left(X_{w}\right)}\right)}
$$

Donde: $\Delta T_{B}$ es el aumento del punto de ebullición, $T$ es el punto de ebullición de la solución, $T_{w}$ es el punto de ebullición del agua pura, $\Delta H_{v}$ es el calor latente de evaporación del agua, $R$ es la constante de los gases ideales y $X_{w}$ es la fracción molar del agua [5].

No obstante, la validez de la ley de Raoult es restringida a soluciones diluidas o ideales, las cuales no son utilizadas en la mayoría de los procesos de interés industrial, haciendo necesario el uso de datos experimentales de la elevación del punto de ebullición en función de la concentración y la presión, los cuales posteriormente se ajustan a modelos matemáticos [6]. Para soluciones muy concentradas, la elevación del punto de ebullición se obtiene mejor a partir de una regla empírica conocida como la regla de Dühring, según la cual la temperatura de ebullición de una determinada solución es una función lineal de la temperatura de ebullición del agua pura a la misma presión [7], la cual es expresada por la Ecuación 2: 


$$
T=m_{0}+m_{1} T_{w}
$$

Donde: $T$ es el punto de ebullición de la solución $\left[{ }^{\circ} \mathrm{C}\right], T_{w}$ es el punto de ebullición del agua pura $\left[{ }^{\circ} \mathrm{C}\right]$, $m_{0}$ y $m_{l}$ son parámetros del modelo característicos de cada solución y se determinan por regresión lineal [6].

Otra manera de representar los datos del punto de ebullición sobre soluciones acuosas se basa en extender el uso de expresiones adecuadas para describir la dependencia de la temperatura con la presión de vapor del agua pura, como es el caso de la ecuación de Antoine [8,9], la cual se escribe como:

$$
\operatorname{Ln}(P)=A-\frac{B}{(T+C)}
$$

Donde: $P$ es la presión [Pa], $T$ es la temperatura de ebullición de la solución [K]; $A, B$, y $C$ son constantes empíricas dependientes en la concentración.

Por otro lado, Crapiste y Lozano propusieron otra alternativa apropiada para la representación de la elevación del punto de ebullición de soluciones acuosas, la cual consiste en adoptar un modelo empírico que es dependiente simultáneamente de la presión y de la concentración de sólidos solubles [8-11], presentando la siguiente ecuación

$$
\Delta T_{B}=\alpha W^{\beta} \exp (\gamma W) P^{\delta}
$$

Donde: $\Delta T_{B}$ es el aumento del punto de ebullición $\left[{ }^{\circ} \mathrm{C}\right], W$ es la concentración de sólidos solubles ['Brix], $P$ es la presión [mbar] y $\alpha, \beta, \gamma$, y $\delta$ son parámetros del modelo que pueden ser evaluados por regresión no lineal.

Estos tres modelos se han aplicado con buen grado de ajuste a datos experimentales de temperaturas de ebullición de algunas matrices alimenticias como jugo de manzana, jugo de caña de azúcar, extracto de café, jugo de toronja, jugo de mora, entre otros [5,6,8-11]. Sin embargo, no se encuentran datos de aumento del punto de ebullición de las pastas de las tres variedades de tomate más usadas por la industria colombiana: Chonto, Milano y Río Grande.

Por tanto, en este trabajo se determinó experimentalmente el aumento del punto de ebullición de pasta de tomate de tres variedades colombianas (Milano, Chonto y Río grande) y se ajustaron los modelos empíricos de Düring, Antoine y Crapiste-Lozano. Estos datos serán de utilidad para la industria de elaboración de pastas y salsa de tomate, ya que podrán mejorar sus procesos al incluir la corrección del aumento del punto de ebullición dentro de sus modelos matemáticos y simulaciones.

\section{Parte experimental}

\section{Materia prima}

Las tres variedades de tomate (Lycopersicon esculentum Mill.) Milano, Chonto y Río Grande fueron adquiridas en el mercado local con las características de grado de color rojo y categoría "extra" descritas en [12] y con las propiedades fisicoquímicas mostradas en la Tabla 1. Los tomates se lavaron, escaldaron en agua a $100^{\circ} \mathrm{C}$ por 3 minutos, despulparon, refinaron y concentraron en un evaporador rotatorio marca Heidolph ${ }^{\circledR}$ (alrededor de $1 \times 10^{4} \mathrm{~Pa}$ absolutos) obteniéndose una pasta concentrada entre 5 y $35^{\circ}$ Brix medidos con un refractómetro marca Bertuchi ${ }^{\circledR}$ a $25^{\circ} \mathrm{C}$ según Método AOAC. 932.12 de 1990 [13]. Cada una de las muestras fue debidamente etiquetada y almacenada a $4^{\circ} \mathrm{C}$ durante 12 horas antes de

\begin{tabular}{|c|c|c|c|}
\hline Análisis & Río Grande & Chonto & Milano \\
\hline Concentración de sólidos solubles $\left({ }^{\circ} \mathrm{Brix}\right)^{\mathrm{a}}$ & $\begin{array}{c}4 \\
\pm 0,10\end{array}$ & $\begin{array}{l}3,5 \\
\pm 0,10\end{array}$ & $\begin{array}{l}3 \\
\pm 0,10\end{array}$ \\
\hline Acidez (porcentaje de ácido cítrico) ${ }^{b}$ & $\begin{array}{r}0,512 \\
\pm 0,01\end{array}$ & $\begin{array}{r}1,173 \\
\pm 0,06\end{array}$ & $\begin{array}{r}0,192 \\
\pm 0,02\end{array}$ \\
\hline $\mathrm{pH}^{\mathrm{c}}$ & $\begin{array}{c}4,31 \\
\pm 0,01\end{array}$ & $\begin{array}{c}4,13 \\
\pm 0,03\end{array}$ & $\begin{array}{c}4,18 \\
\pm 0,01\end{array}$ \\
\hline Humedad (porcentaje en base húmeda) ${ }^{d}$ & $\begin{array}{r}95,22 \\
\pm 0,05\end{array}$ & $\begin{array}{r}96,67 \\
\pm 0,12\end{array}$ & $\begin{array}{l}96,87 \\
\pm 0,67\end{array}$ \\
\hline
\end{tabular}
realizar los ensayos.

Tabla 1. Análisis fisicoquímico de pulpas de tomate (variedades Río Grande, Chonto y Milano).

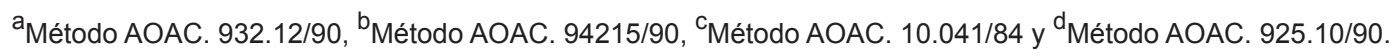




\section{Equipo de medición de la temperatura de ebullición}

En la Figura 1 se muestra un diagrama que describe al equipo utilizado para las medidas experimentales, similar al utilizado por Telis-Romero $\mathrm{J}$ [9]. El equipo consistió de un balón de vidrio fondo plano $(F)$ con tres bocas calentado en un baño de aceite con temperatura controlada (130 \pm $1^{\circ} \mathrm{C}$ ). La temperatura de ebullición se midió con un termómetro digital con una precisión de $\pm 0,01^{\circ} \mathrm{C}$. El vacío del sistema se realizó a través de una bomba de vacío (marca Riestchle ${ }^{\circledR}$ ) conectada con el condensador y la presión fue medida con un sistema de medición de presión digital con una precisión de $\pm 0,01 \mathrm{mbar}$.

La calibración del equipo se determinó con agua destilada a las diferentes presiones utilizadas en este estudio. Para comprobar dicha calibración se utilizó la prueba de comparación de medias entre los datos experimentales y los datos de tablas de vapor saturado reportados en la literatura [14], encontrándose un valor $p$ de 0,7942 que indicaba que el equipo permitía una buena reproducibilidad de los datos.

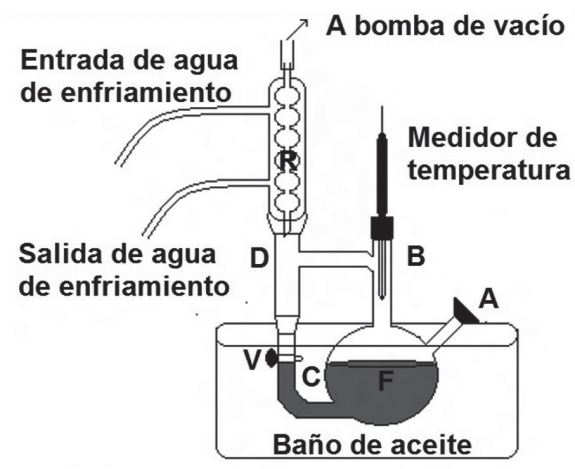

Figura 1. Esquema del equipo utilizado para las mediciones experimentales.

\section{Diseño experimental}

El experimento fue llevado a cabo por triplicado con muestras en el balón de ebullición de $250 \mathrm{ml}$ de pasta de tomate de tres variedades colombianas (Milano, Chonto y Río Grande). Las concentraciones de sólidos solubles de las pastas utilizadas fueron $\{5,11,17,23,29,35\}^{\circ}$ Brix y las presiones absolutas manipuladas en el equipo fueron $\{949,3$; 849,3; 749,3; 649,3; 549,3; 449,3; $349,3 ; 249,3 ; 149,3 ; 49,3\}$ mbar.

\section{Análisis estadístico y procesamiento de datos}

Se realizaron análisis de varianzas $(p<0,05)$, ajuste de regresiones lineales (para los modelo de Dühring y Crapiste-Lozano linealizado) y no lineales (para el modelo de Antoine), usándose el programa Statgraphics Centurion XV versión de 2007.

\section{Resultados y Discusión}

En la Tabla 2 se presenta el Análisis de Varianza para los datos experimentales de temperatura de ebullición de las pastas de tomate. Esta tabla descompone la variabilidad de la Temperatura de ebullición en las contribuciones debidas a varios factores y sus respectivas interacciones. Puesto que se ha elegido la suma de cuadrados Tipo III, se ha medido la contribución de cada factor eliminando los efectos del resto de los factores, así como el efecto de la interacción entre los mismos. Debido a que todos los valores $p$ son inferiores a 0,05 , la concentración, la presión, la variedad de tomate y sus interacciones tienen efecto estadísticamente significativo en la temperatura de ebullición de las pastas de tomate.

Tabla 2. Análisis de la Varianza para Temperatura de ebullición-Suma de Cuadrados Tipo III.

\begin{tabular}{lccccc}
\hline \multicolumn{1}{c}{ Fuente } & $\begin{array}{c}\text { Suma de } \\
\text { Cuadrados }\end{array}$ & GL & Cuadrado Medio & Razón-F & Valor-p \\
\hline Concentración & 52,3612 & 5 & 10,4722 & 169,87 & 0,0000 \\
Presión & 156399 & 9 & 17377,7 & 281885 & 0,0000 \\
Variedad & 76,349 & 2 & 38,1745 & 619,23 & 0,0000 \\
& & & & & \\
Concentración* Presión & 14,9768 & 45 & 0,332817 & 5,40 & 0,0000 \\
Concentración* Variedad & 8,17852 & 10 & 0,817852 & 13,27 & 0,0000 \\
Presión *Variedad & 20,5717 & 18 & 1,14287 & 18,54 & 0,0000 \\
Concentración* Presión* Variedad & 13,823 & 90 & 0,153588 & 2,49 & 0,0000 \\
& & & & & \\
Residuos & 22,1933 & 360 & 0,0616481 & & \\
Total (corregido) & 156608 & 539 & & & \\
\hline
\end{tabular}




\section{Evaluación del modelo de Dühring}

Las Figuras 2, 3 y 4 muestran los valores de la temperatura de ebullición de las pastas de tomates de las diferentes variedades y concentraciones de soluto frente a las temperaturas de ebullición del agua pura. Se observa que existe una desviación positiva entre estos dos valores, definida como el aumento del punto de ebullición de una solución. Este efecto se debe a que cuando un soluto no volátil se encuentra disuelto en el agua, la presencia de las moléculas del soluto reducen la capacidad de las moléculas del solvente a pasar de la fase líquida a la fase de vapor por la generación de nuevas fuerzas de interacción que producen un desplazamiento del equilibrio, lo que se traduce en una reducción de la presión de vapor sobre la solución afectando de esta manera el punto de ebullición [15]. Para temperaturas altas, esta desviación es pequeña y prácticamente las líneas de tendencia entre las diferentes concentraciones tienen la misma pendiente y para temperaturas bajas la desviación es mayor con diferencias marcadas entre las pendientes de las líneas de tendencia. Estas discrepancias son debidas al efecto que produce la interacción de las presiones bajas con las altas concentraciones de soluto $[15,16]$.

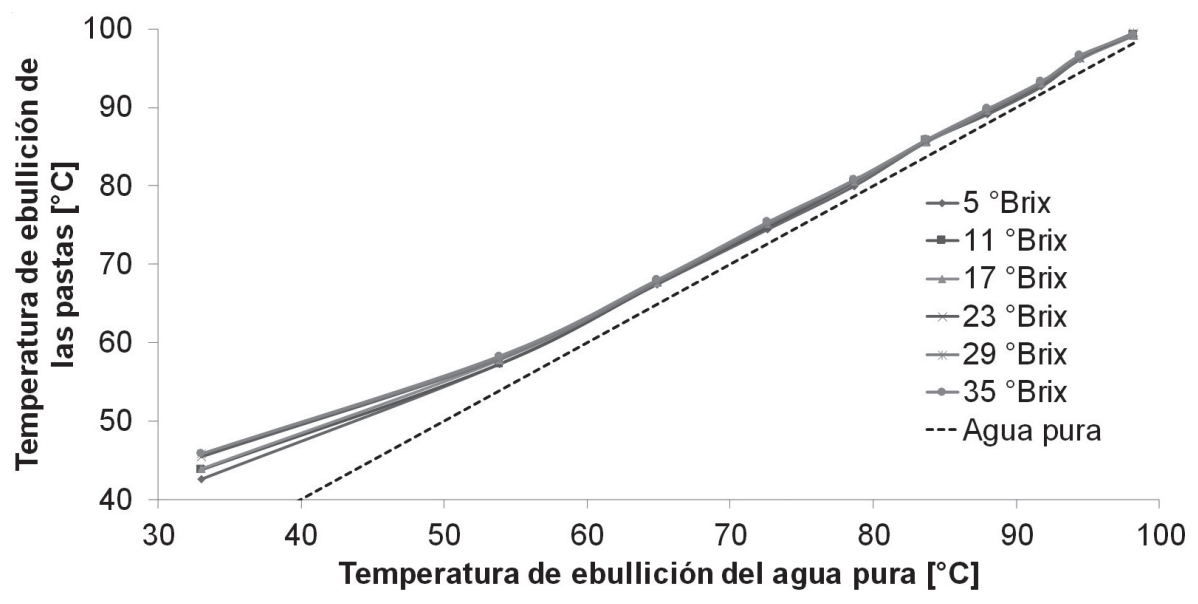

Figura 2. Relación entre la temperatura de ebullición del agua pura y la temperatura de ebullición de la pasta de tomate variedad Río Grande.

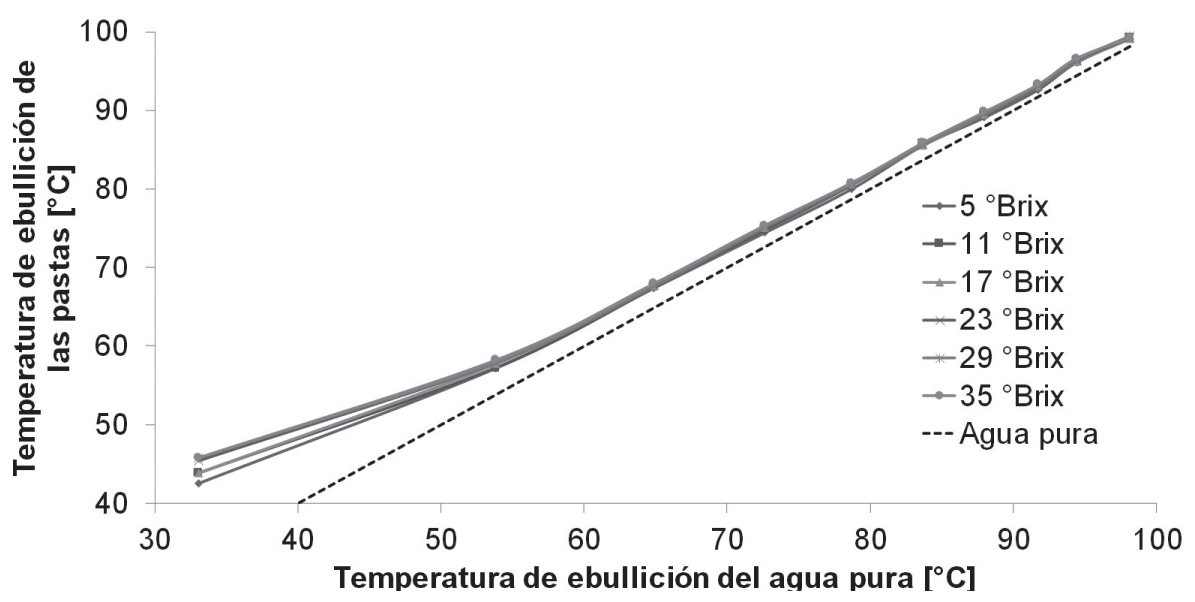

Figura 3. Relación entre la temperatura de ebullición del agua pura y la temperatura de ebullición de la pasta de tomate variedad Chonto. 


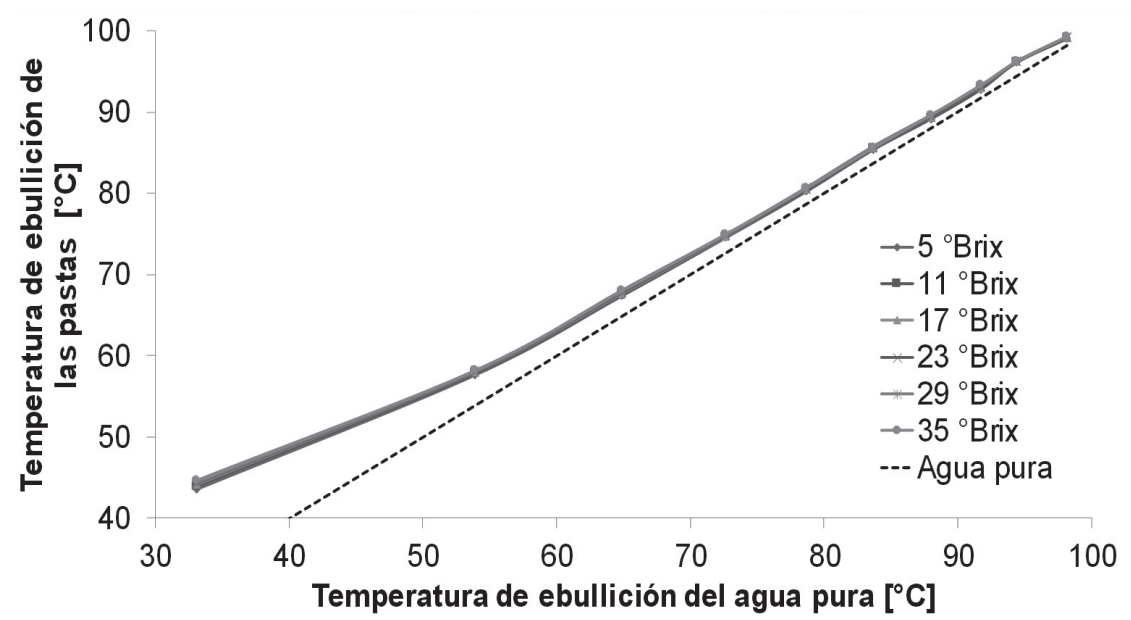

Figura 4. Relación entre la temperatura de ebullición del agua pura y la temperatura de ebullición de la pasta de tomate variedad Milano.

Si en la Ecuación 2 se iguala el valor de la pendiente $\left(m_{1}\right)$ a 1 , se tendría que $\Delta T_{B}$ es igual a $m_{0}$, indicando que bajo esta condición la elevación del punto de ebullición varía solamente con la concentración de la solución y es independiente de la presión $[9,17,18]$. En la Tabla 3 se muestran los valores de los parámetros del modelo de Dühring para cada variedad y los valores de $r^{2}$, que indican un buen ajuste del modelo a los datos experimentales, además se observa que los valores de $m_{l}$, en todos los casos, son menores que 1 y van disminuyendo a medida que aumenta la concentración de sólidos solubles de las pastas de tomate de las tres variedades estudiadas, lo cual implica que la elevación del punto de ebullición no solo se ve influenciada por la concentración sino también por la presión ejercida sobre las muestras. Comportamientos similares se presentaron para soluciones de extracto de café [9] y para soluciones de jugo de uva a altas concentraciones [10], mientras que para soluciones modelo de caña de azúcar $m_{1}$ fue prácticamente igual a 1 para la diferentes concentraciones [6].

Tabla 3. Parámetros estimados del modelo de Dühring.

\begin{tabular}{|c|c|c|c|c|c|c|c|}
\hline \multirow[b]{2}{*}{ Variedad } & \multirow[b]{2}{*}{ Parámetro } & \multicolumn{6}{|c|}{ Concentración [ ${ }^{\circ}$ Brix] } \\
\hline & & 5 & 11 & 17 & 23 & 29 & 35 \\
\hline \multirow{3}{*}{ Río grande } & $m_{0}$ & 8,7825 & 11,0162 & 10,1647 & 11,2388 & 11,1292 & 11,8303 \\
\hline & $m_{1}$ & 0,9014 & 0,8815 & 0,8941 & 0,8840 & 0,8873 & 0,8796 \\
\hline & $r^{2}$ & 0,9974 & 0,9955 & 0,9971 & 0,9969 & 0,9975 & 0,9972 \\
\hline \multirow{3}{*}{ Chonto } & $m_{0}$ & 10,9432 & 12,0412 & 12,4912 & 14,0889 & 14,2883 & 14,4919 \\
\hline & $m_{1}$ & 0,8888 & 0,8786 & 0,8741 & 0,8562 & 0,8554 & 0,8536 \\
\hline & $r^{2}$ & 0,9977 & 0,9967 & 0,9973 & 0,9957 & 0,9953 & 0,9956 \\
\hline \multirow{3}{*}{ Milano } & $m_{0}$ & 12,1814 & 12,5194 & 12,8073 & 12,9596 & 13,2195 & 13,4012 \\
\hline & $m_{1}$ & 0,8748 & 0,8720 & 0,8699 & 0,8687 & 0,8672 & 0,8654 \\
\hline & $r^{2}$ & 0,9971 & 0,9970 & 0,9970 & 0,9970 & 0,9970 & 0,9968 \\
\hline
\end{tabular}

\section{Evaluación del modelo de Antoine}

Las Figuras 5, 6 y 7 muestran los valores de la temperatura de ebullición frente a la presión de vapor de las pastas de tomates de las diferentes variedades. Se observa que existe un comportamiento exponencial descrito por la ecuación de Clapeyron [14] y que las curvas de las pastas están desviadas a la derecha con respecto a la curva del agua pura, lo cual se debe a la disminución de la presión de vapor por efecto del soluto no volátil. Además, para cada concentración de la pasta de tomate, se observa que a medida que la presión disminuye el aumento del punto de ebullición es más grande, esto se debe a que el comportamiento de la presión de vapor por efecto de la temperatura presenta un comportamiento no lineal a presiones bajas, mientras que a presiones altas su comportamiento 
es casi lineal. Por otro lado, se observa que a una concentración y presión determinadas, el aumento del punto de ebullición es más pequeño para la variedad Río Grande comparada con las variedades Chonto y Milano, esto se debe al efecto de los diferentes tipos de solutos que conforman la pasta siendo los solutos con mayor peso molecular (disacáridos, ácidos, pectinas) los causantes de los mayores aumentos en el punto de ebullición [16].

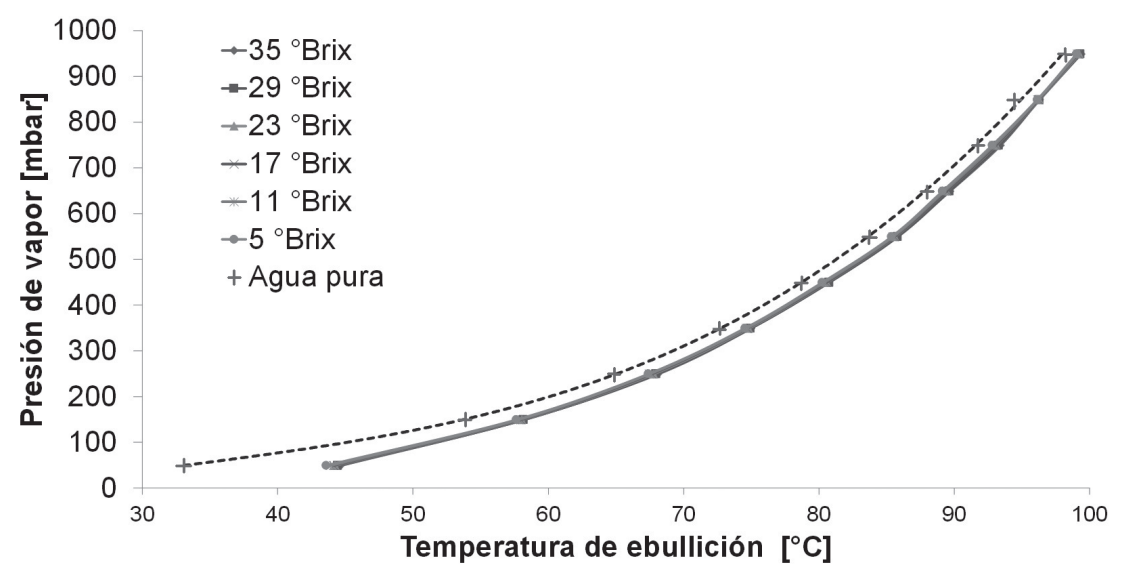

Figura 5. Relación entre la temperatura de ebullición y presión de vapor de la pasta de tomate Milano.

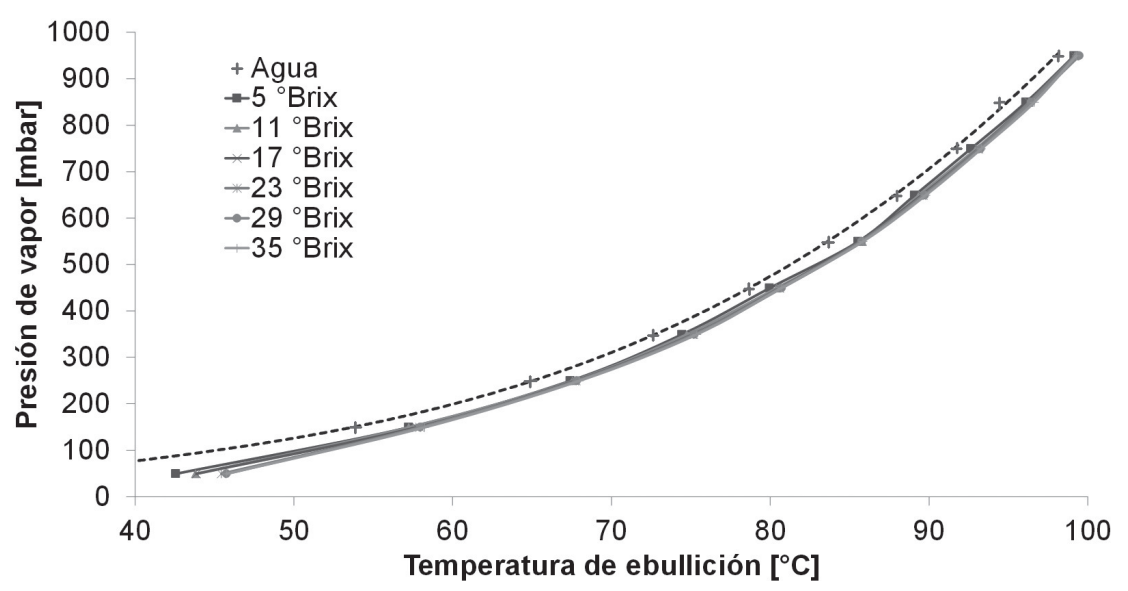

Figura 6. Relación entre la temperatura de ebullición y presión de vapor de la pasta de tomate variedad Chonto.

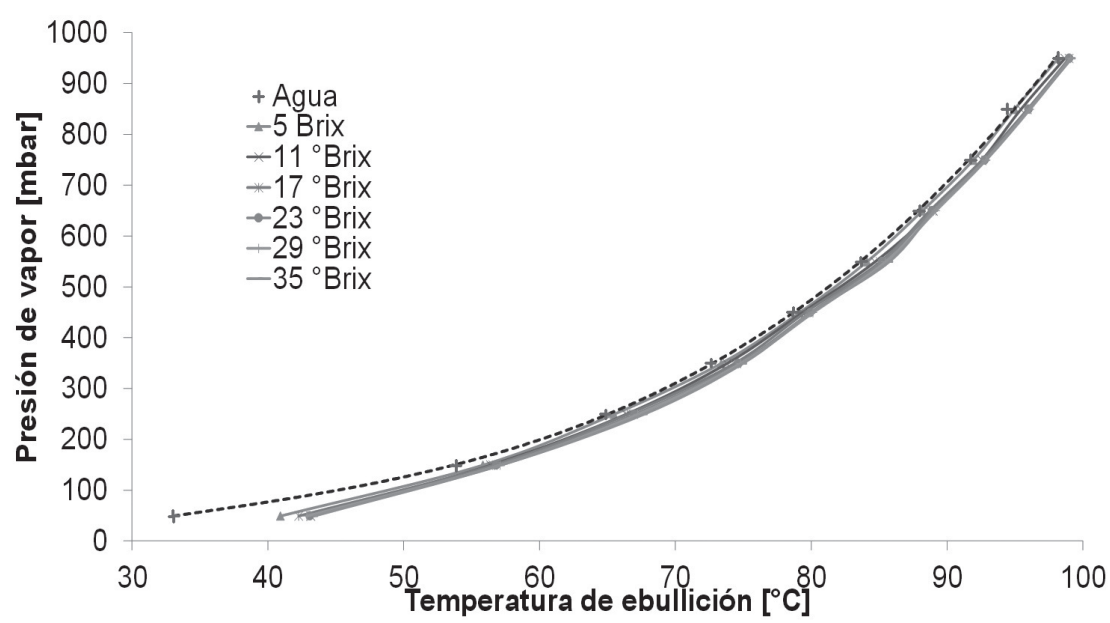

Figura 7. Relación entre la temperatura de ebullición y presión de vapor de la pasta de tomate variedad Río Grande. 
En la Tabla 4 se muestran las constantes estimadas $(A, B$ y $C)$ para la ecuación de Antoine ( $P: P a$ y $T: K)$ así como el coeficiente de determinación y la sumatoria del cuadrado del error (SCE) que indica un buen ajuste del modelo a los datos experimentales. Los valores de las constantes $A, B$ y $C$ son muy similares para las tres variedades y para las diferentes concentraciones de sólidos solubles, por lo cual no es posible establecer una dependencia explícita de estas constantes como una función del contenido de sólidos solubles presentes en los tomates, lo cual es similar a lo reportado para el jugo de uva [10].

Tabla 4. Parámetros estimados para el modelo de Antoinne.

\begin{tabular}{|c|c|c|c|c|c|c|c|}
\hline \multirow{2}{*}{ Variedad } & \multirow{2}{*}{ Parámetro } & \multicolumn{6}{|c|}{ Concentración [ ${ }^{\circ}$ Brix] } \\
\hline & & 5 & 11 & 17 & 23 & 29 & 35 \\
\hline \multirow{5}{*}{ Río grande } & $A$ & 19,2206 & 18,6733 & 18,7286 & 17,7883 & 19,1026 & 17,8463 \\
\hline & $B$ & 1689,30 & 1473,28 & 1496,81 & 1147,37 & 1616,47 & 1156,15 \\
\hline & $C$ & $-153,674$ & $-167,702$ & $-166,222$ & $-191,007$ & $-160,798$ & $-191,368$ \\
\hline & $r^{2}$ & 0,9992 & 0,9976 & 0,9994 & 0,9994 & 0,9996 & 0,9990 \\
\hline & SCE & 8,66 & 15,31 & 8,99 & 5,21 & 8,49 & 5,02 \\
\hline \multirow{4}{*}{ Chonto } & $A$ & 18,8955 & 17,9039 & 19,2234 & 18,8911 & 18,4451 & 18,8059 \\
\hline & $B$ & 1540,99 & 1177,99 & 1642,63 & 1508,41 & 1353,07 & 1475,40 \\
\hline & $C$ & $-165,042$ & $-189,761$ & $-160,802$ & $-169,462$ & $-178,973$ & $-171,776$ \\
\hline & $r^{2}$ & 0,9995 & 0,9991 & 0,9996 & 0,9994 & 0,9992 & 0,9993 \\
\hline \multirow{6}{*}{ Milano } & SCE & 6,57 & 6,33 & 9,90 & 16,62 & 16,28 & 16,76 \\
\hline & $A$ & 18,7118 & 18,8037 & 18,9301 & 18,7689 & 17,9917 & 18,7245 \\
\hline & $B$ & 1460,42 & 1491,11 & 1534,66 & 1473,19 & 1193,36 & 1453,62 \\
\hline & $C$ & $-170,882$ & $-169,286$ & $-166,963$ & $-170,907$ & $-189,911$ & $-172,492$ \\
\hline & $r^{2}$ & 0,9996 & 0,9996 & 0,9996 & 0,9996 & 0,9994 & 0,9995 \\
\hline & SCE & 8,53 & 9,68 & 10,52 & 9,49 & 5,21 & 10,20 \\
\hline
\end{tabular}

\section{Evaluación del modelo de Crapiste y Lozano} En la Tabla 5 se muestran los parámetros estimados de la ecuación de Crapiste y Lozano y el coeficiente de determinación que indica un buen ajuste del modelo a los datos experimentales. Se observa que los valores de $\beta$ son similares a los reportados para el jugo de uva $(\beta=1,2851)$ [10], pero $\beta$ y las demás constantes difieren en orden de magnitud a lo reportado para los extractos de café [9], jugo de mandarina [19], jugo de uva [10] y soluciones modelo de sacarosa [6]. Estas marcadas diferencias se deben a la contribución que presentan los diferentes componentes de las pastas de tomate (ácidos, azúcares, minerales, entre otros) en la elevación del punto de ebullición y a la hidrólisis de los azúcares durante el calentamiento debido al $\mathrm{pH}$ ácido de la pasta (entre 4,13 y 4,31 ) $[9,15]$.

Tabla 5. Parámetros estimados para el modelo de Crapiste y Lozano.

\begin{tabular}{cccc}
\hline Parámetro & Rio grande & Chonto & Milano \\
\hline$\alpha$ & 6,39956 & 7,03450 & 8,27729 \\
$\beta$ & 1,42720 & 1,39560 & 1,35786 \\
$\gamma$ & $-0,05563$ & $-0,05675$ & $-0,06283$ \\
$\delta$ & $-0,713388$ & $-0,655664$ & $-0,642599$ \\
$r^{2}$ & 0,9852 & 0,9662 & 0,9856 \\
\hline
\end{tabular}




\section{Conclusiones}

La concentración, la presión, la variedad de tomate y las diferentes interacciones de estas variables tuvieron efecto estadísticamente significativo al $5 \%$ de significancia en la temperatura de ebullición de las pastas de tomate.

La temperatura de ebullición de la pasta de tomate fue más alta que la temperatura de ebullición del agua pura a la misma presión y concentración, siendo más grande la diferencia entre los valores cuando la concentración es más alta.

Se observó que a una concentración y presión determinadas, el aumento del punto de ebullición es más pequeño para la variedad Río Grande comparada con las variedades Chonto y Milano.

Los datos experimentales del aumento del punto de ebullición de la pasta de tomate fueron ajustados por los modelos de Dühring, Antoine y Crapiste-Lozano. Sin embargo, no fue posible establecer una dependencia de las constantes de la ecuación de Antoine como una función de los sólidos solubles totales de la pulpa de tomate debido a su similitud en los valores.

\section{Referencias bibliográficas}

[1] SIPSA. Boletín mensual \#8. Insumos y factores asociados a la producción agropecuaria. Bogotá, Colombia: DANE y Ministerio de Agricultura; 2013.

[2] Pinzón M, Cardozo C, Portilla M. Evaluación de las propiedades fisicoquímicas en el proceso de maduración del tomate cv. Milano producido a campo abierto y bajo invernadero. @limentech. 2013;11(1):22-30.

[3] Levine, I. Fisicoquímica. Volumen I. 5 ed. Madrid: McGraw-Hill editores; 2004.

[4] Westphalen D. Cálculo da elevação do ponto de ebulição de soluções a partir de dados de pressão parcial (sitio en internet). Escola de Engenharia Mauá-IMT. Disponible en: www. hottopos.com.br/regeq6/denis.htm. Acceso el 21 de Agosto de 2014.

[5] Crapiste G, Lozano J. Effect of Concentration and Pressure on the Boiling Point Rise of Apple Juice and Related Sugar Solutions. J. Food Sci. 1988;53:865-9.

[6] Montes E, Torres R, Andrade R. Aumento del punto de ebullición de soluciones modelos para jugo de caña de azúcar. Temas Agrarios.
2006;11(2):5-13.

[7] McCabe W, Smith J, Harriott P. Operaciones Unitarias en ingeniería Química. 7 ed. México D.F.: McGraw-Hill editores; 2007.

[8] Montes E, Torres R, Andrade R. Ecuación de Crapiste y Lozano modificada para predecir la elevación en la temperatura de ebullición en soluciones de caña de azúcar. Dyna. 2007;74(153):135-40.

[9] Telis-Romero J, Cabral R, Kronka G. Elevation of boiling point coffee extract. Braz J Chem Eng. 2002;19(1):119-26.

[10] Telis-Romero J, Cantú-Lozano D, Telis V, Gabas A. Thermal Evaporation: Representation of rise in boiling point of grapefruit juice. Food Sci Technol Int. 2007;13(3):225-9.

[11] Gabas A, Sobral P, Cardona C, Telis V, TelisRomero J. Influence of fluid concentration on the elevation of boiling point of blackberry juice. Int J Food Prop. 2008;11(4):865-75.

[12] ICONTEC. Norma Técnica Colombiana. NTC 1103-1. Industrias alimentarias. Tomate de mesa. Bogotá, Colombia: Instituto Colombiano de Normas Técnicas y Certificación; 1995.

[13]A.O.A.C. Official Methods of Analysis of the Association of Official Analytical Chemist. Washington D.C., USA: Association of Official Analytical Chemists; 1990.

[14] Smith J, Van Ness H, Abbott M. Introduction to Chemical Engineering Thermodynamics. 7 ed. México D.F.: McGraw-Hill editores; 2005.

[15]Armas V, Lezama R, Iparraguirre R, Corcuera A, Siche R. Aumento ebulliscópico de extracto de jugo de yacón (Smallanthus sonchifolius) y determinación de gráficas de Dühring. Agroind Sci. 2012;2:146-52.

[16]Devahastin S. Physicochemical aspects of Food Engineering and processing. Florida: CRC Press; 2011.

[17]Maximo G, Meirelles A, Batista E. Boiling point of aqueous d-glucose and d-fructose solutions: Experimental determination and modeling with group-contribution method. Fluid Phase Equilibr. 2012;299:32-41.

[18] Varshney N, Barhate V. Effect of Concentrations and Vacua on Boiling Points of Fruit Juices. J Food Tech. 1978;13(3):225-33.

[19] llangantileke S, Ruba A, Joglekar H. Boiling point rise of concentrated Thai Tangerine juices. J Food Eng. 1991;15(1):235-43. 